\title{
Teambesprechungen planen und durchführen
}

\begin{abstract}
In vielen Arztpraxen sind regelmäßige Besprechungen zwischen Arzt und Praxisteam eher die Ausnahme als die Regel. Oft herrscht die Auffassung, dass solche Meetings zu viel Zeit in Anspruch nehmen und Fragen auch zwischendurch angesprochen und geklärt werden können. Dabei ist bei den Mitarbeitern fast immer ein Bedürfnis nach separaten Teambesprechungen vorhanden.
\end{abstract}

Bei der Planung einer Teambesprechung sollten zunächst die räumlichen und zeitlichen Rahmenbedingungen festgelegt werden. Ein Zeitrahmen von einer Stunde ist in der Regel ausreichend. Wenn in der Praxis kein Besprechungszimmer vorhanden ist, kann der Wartebereich für die Teambesprechung genutzt werden. Der Vorteil hierbei ist, dass der Arzt nicht hinter seinem Schreibtisch sitzt und während des Gesprächs durch Telefonanrufe oder Ähnliches abgelenkt wird. Zudem fördert die "Chefposition" hinter dem Schreibtisch das Untergebenengefühl der Mitarbeiter und wirkt so einer entspannten, ehrlichen Diskussion mit einfallsreichen Ideen entgegen. Mitarbeiter neigen in so einer Situation eher dazu, auch den für sie ungeeignet erscheinenden Vorschlägen schnell zu zustimmen. An Absprachen, die unter dieser Voraussetzung getroffen werden, wird sich in der Folge meist nur unzureichend gehalten. Zur inhaltlichen Vorbereitung einer Teambesprechung hat sich in vielen Arztpraxen das folgende System bewährt: Jeder, der einen Punkt besprechen möchte, schreibt inn auf einen Zettel und legt diesen in eine Teambesprechungsmappe oder -box. Der für die Teambesprechung verantwortliche Mitarbeiter leert die Box vor Beginn der Sitzung, schreibt die Inhalte in Stichpunkten auf neutrale Zettel und bringt sie zur Teambesprechung mit.

\section{Ablauf einer Teambesprechung}

Damit das gesamte Team motiviert in die Besprechung geht, empfiehlt es sich, gewisse „Spielregeln“ aufzustellen, wie zum Beispiel:

- Jeder fasst sich kurz.

- Man fällt sich gegenseitig nicht ins Wort.

- Jeder sagt seine Meinung, auch die Auszubildenden.

- Es muss sachlich und fair argumentiert werden.

- Persönliche Konflikte werden nicht vor der Gruppe ausgetragen.

- Eine entspannte Atmosphäre ist erwünscht; Lachen ist erlaubt.

Zu Beginn der Teambesprechung nimmt nun jeder einen Zettel aus der Teambesprechungsbox, öffnet inn und liest das Thema laut vor. Der Vorteil dieser Methode ist, dass man so nicht unbedingt die eigenen Punkte vorträgt bzw. verteidigt und damit offener für die Themen der anderen ist. Wer das Thema vorliest, macht es zu seinem Anliegen.
Bei einer Teambesprechung kann es verschiedene Rollen geben, die bei jedem Meeting neu verteilt werden sollten: den Moderator, den Schriftführer und das "Gewissen“. Der Moderator organisiert und moderiert das Meeting. Der Schriftführer schreibt und verteilt das Protokoll. Das "Gewissen“ hat das letzte Wort. Es sagt jedem Einzelnen, in neutraler und konfliktfreier Form, was gut und was schlecht gelaufen ist. So wird ein Lerneffekt generiert und jeder dazu angespornt, es beim nächsten Mal besser zu machen. Die wechselnden Rollen geben jedem im Team Verantwortung für den Erfolg der Meetings und entlasten den Arzt. Dieser sollte jedoch weiterhin bei wichtigen Entscheidungen von seinem Primus-interpares- oder Vetorecht Gebrauch machen. Bei manchen Themen ist die Anwesenheit des Chefs allerdings gar nicht erforderlich, oder er kann nur als Zuhörer und nicht als Vorgesetzter an der Gesprächsrunde teilnehmen. Das lockert das Klima auf und bringt dem Arzt erfahrungsgemäß neue Einsichten.

Wenn alle Besprechungspunkte bearbeitet und die Lösungen klar definiert sind, werden diese schriftlich in einem Protokoll festgehalten. Im Protokoll wird schriftlich dokumentiert:

\footnotetext{
- wer anwesend war,

- was besprochen bzw. entschieden wurde und

- was bis zu welchem Zeitpunkt von wem erledigt werden soll.
}

Am Ende des Meetings wird das Protokoll vorgelesen, von den anwesenden Mitarbeitern unterschrieben und in einem Besprechungsordner abgelegt, zu dem alle Mitarbeiter Zugriff haben.

\section{Korrespondenzadresse \\ Monika Dumont \\ Leitung Kommunikation und Marketing \\ Duxxess - Gesellschaft für medizinisches Erfolgsmanagement mbH monika.dumont@duxxess.com}

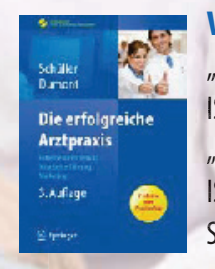

Weiterführende Literatur:

"Die erfolgreiche Arztpraxis",

ISBN 978-3-642-00733-0 und

"Unternehmen Arztpraxis - Ihr Erfolgsmanagement",

ISBN 978-3-540-48559-9

Springer Verlag, 2009, je 39,95€ 